\title{
Correction to: $\mathrm{A} \mathrm{CCR5}^{+}$memory subset
} within HIV-1-infected primary resting $\mathrm{CD}^{+} \mathrm{T}$ cells is permissive for replication-competent, latently infected viruses in vitro

Kazutaka Terahara ${ }^{1 *}$, Ryutaro Iwabuchi ${ }^{1,2,3}$, Masahito Hosokawa ${ }^{4,5}$, Yohei Nishikawa ${ }^{2,3}$, Haruko Takeyama ${ }^{2,3,4,5}$, Yoshimasa Takahashi ${ }^{1}$ and Yasuko Tsunetsugu-Yokota, ${ }^{1,6}$

\section{Correction to: BMC Res Notes (2019) 12:242} https://doi.org/10.1186/s13104-019-4281-5

After publication of the original article [1], the authors became aware of a miscalculation in the original Fig. $2 \mathrm{~d}$.

$\% \mathrm{HIV}-1^{+}$activated cells at Day $5-\% \mathrm{HIV}-1^{+}$resting cells at Day 5

$$
\% \text { HIV-1 }{ }^{+} \text {activated cells at Day } 5
$$

should be calculated as:

$\frac{\% \mathrm{HIV}-1^{+} \text {activated cells at Day } 5-\% \mathrm{HIV}-1^{+} \text {resting cells at Day } 5}{\% \mathrm{HIV}-1^{+} \text {resting }} \times 100$

The corrected Fig. $2 \mathrm{~d}$ is shown in this erratum.

*Correspondence: tera@nih.go.jp

${ }^{1}$ Department of Immunology, National Institute of Infectious Diseases,

1-23-1 Toyama, Shinjuku-ku, Tokyo 162-8640, Japan

Full list of author information is available at the end of the article 
a

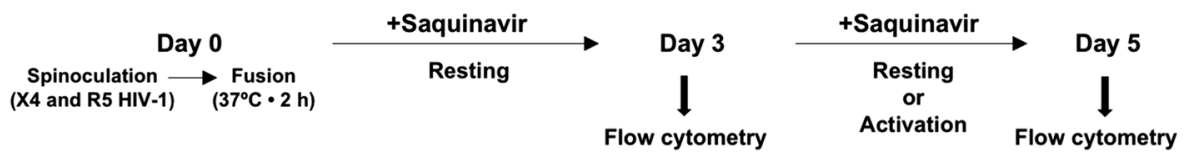

b
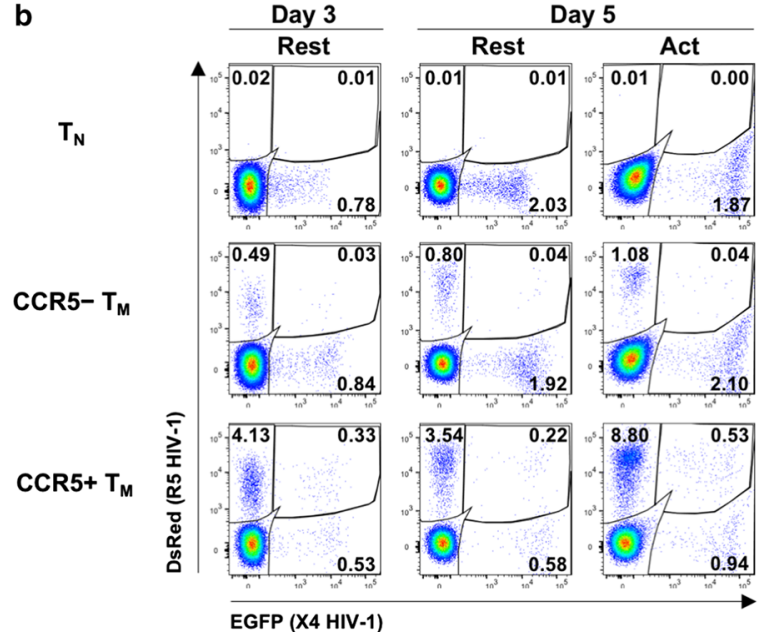

Day 5

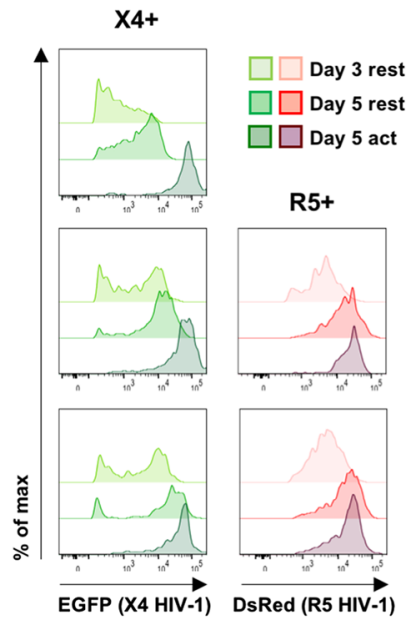

C

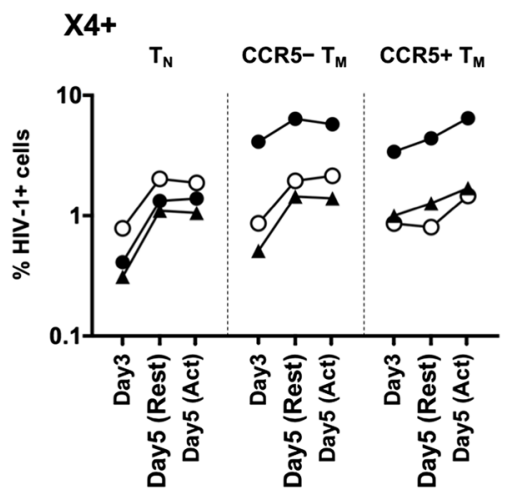

R5+
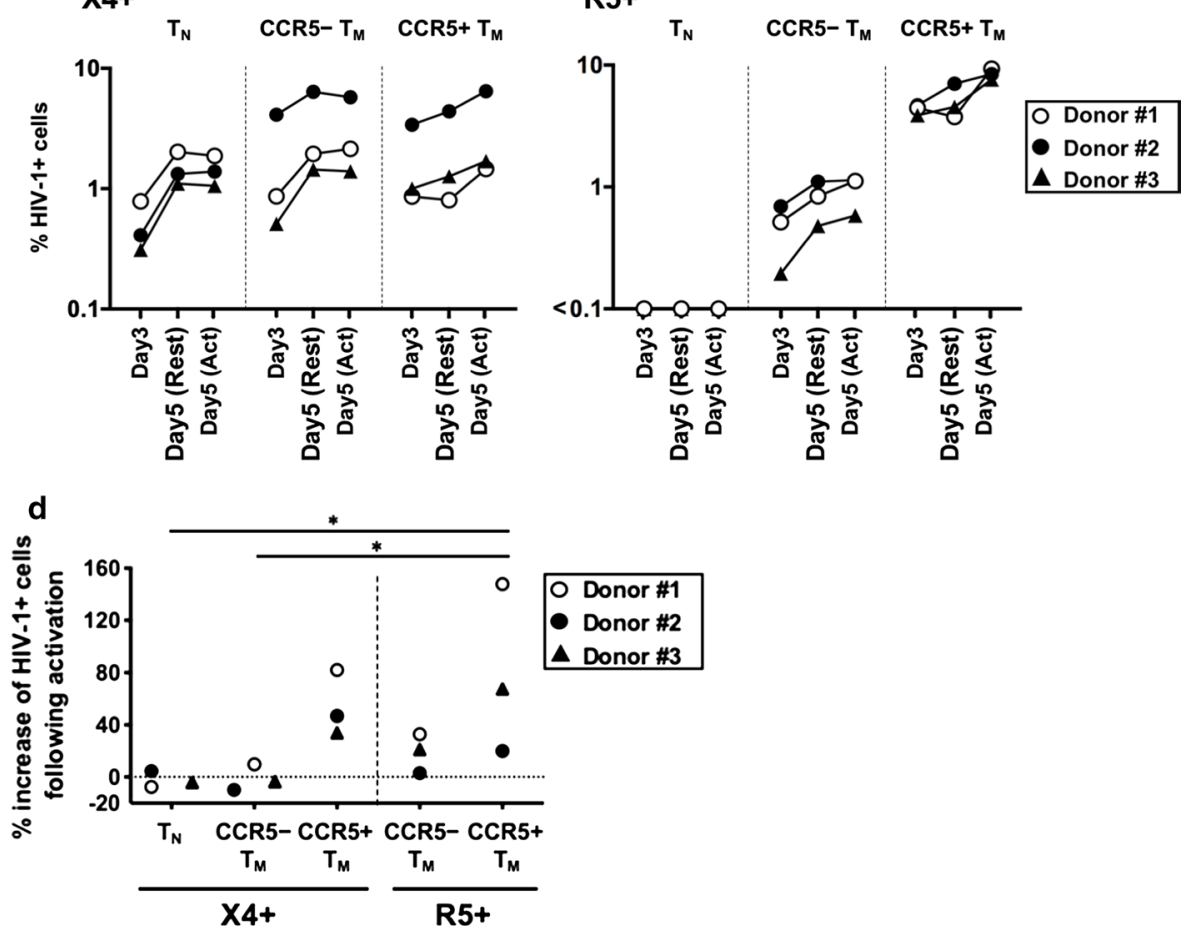

Fig. 2 HIV-1 infection and culture of resting $C D 4^{+}$T-cell subsets isolated by cell sorting. Subsets of naïve $T$ cells $\left(T_{N}\right)$, or $C C R 5^{+}$or $C C R 5^{-}$memory T cells $\left(T_{M}\right)$, were separately infected and cultured. a Schematic of the protocol of HIV-1 infection and culture. $\mathbf{b}$ Representative flow-cytometry profiles of cells from Donor \#1 at day 3 and day 5 post-infection (resting or activated), separated according to reporter expression indicating the presence of X4 or R5 HIV-1, with the percentage of each subset indicated (left panels). The intensity of fluorescence for each viral reporter in each cell subset [except for the very low percentage of DsRed ${ }^{+}$cells $\left(R 5^{+}\right)$in $T_{N}$ cells] is shown in the right-hand panels. c Percentages of HIV- $1^{+}$cells in each $\mathrm{CD} 4^{+}$T-cell subset in three donors. $\mathbf{d}$ Percentage increases in frequencies of HIV-1 ${ }^{+}$cells following activation were estimated by comparing percentages of $\mathrm{HIV}_{-} 1^{+}$cells in the activation condition with those in the resting condition at day 5 post-infection. Significant differences $\left({ }^{*} P<0.05\right.$, ${ }^{*} P<0.01$ ) were determined by repeated-measures one-way ANOVA followed by Tukey's multiple comparison test. In $\mathbf{c}$ and $\mathbf{d}, \mathrm{HIV}-1^{+}$cells include the corresponding reporter (either EGFP or DsRed) single-positive cells and double-positive cells 
Although the statistical significances have been altered, the hierarchical mode between cell-subset groups remains the same. It is still shown that numbers of $\mathrm{X} 4$ $\mathrm{HIV}-1^{+}$cells increased consistently in the CCR $5^{+} \mathrm{TM}$ subset of all three donors tested. Therefore, the correction does not change the scientific conclusion.

\section{Author details}

${ }^{1}$ Department of Immunology, National Institute of Infectious Diseases, 1-23-1 Toyama, Shinjuku-ku, Tokyo 162-8640, Japan. ${ }^{2}$ Department of Life Science and Medical Bioscience, Waseda University, 2-2 Wakamatsu-cho, Shinjuku-ku, Tokyo 162-8480, Japan. ${ }^{3}$ Computational Bio Big-Data Open Innovation Laboratory, National Institute of Advanced Industrial Science and Technology, 3-4-1 Okubo, Shinjuku-ku, Tokyo 169-8555, Japan. ${ }^{4}$ Research Organization for Nano \& Life Innovation, Waseda University, 513 Wasedatsurumaki-cho, Shinjuku-ku, Tokyo 162-0041, Japan. ${ }^{5}$ Institute for Advanced Research of Biosystem

Dynamics, Waseda Research Institute for Science and Engineering, Waseda University, 2-2 Wakamatsu-cho, Shinjuku-ku, Tokyo 162-8480, Japan. ${ }^{6}$ Department of Medical Technology, School of Human Sciences, Tokyo University of Technology, 5-23-22 Nishikamata, Ota-ku, Tokyo 144-8535, Japan.
The original article can be found online at https://doi.org/10.1186/s1310 4-019-4281-5.

Received: 4 June 2019 Accepted: 4 June 2019

Published online: 10 June 2019

\section{Reference}

1. Terahara K, Iwabuchi R, Hosokawa M, Nishikawa Y, Takeyama H, Takahashi Y, Tsunetsugu-Yokota Y. A CCR5 ${ }^{+}$memory subset within HIV-1-infected primary resting $\mathrm{CD}^{+}{ }^{+} \mathrm{T}$ cells is permissive for replication-competent, latently infected viruses in vitro. BMC Res Notes. 2019;12:242. https://doi. org/10.1186/s13104-019-4281-5.

\section{Publisher's Note}

Springer Nature remains neutral with regard to jurisdictional claims in published maps and institutional affiliations. 